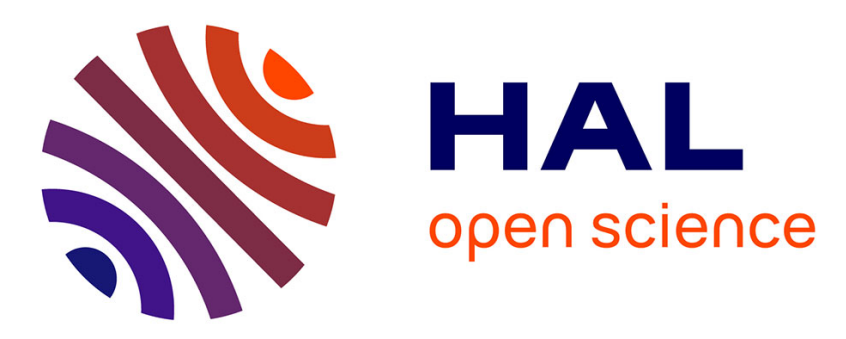

\title{
La radioactivité alpha étudiée par les méthodes de la fission
}

\author{
D.N. Poenaru, M. Ivaşcu, A. Sândulescu
}

\section{To cite this version:}

D.N. Poenaru, M. Ivaşcu, A. Sândulescu. La radioactivité alpha étudiée par les méthodes de la fission. Journal de Physique Lettres, 1979, 40 (18), pp.465-467. 10.1051/jphyslet:019790040018046500 . jpa00231666

\section{HAL Id: jpa-00231666 https://hal.science/jpa-00231666}

Submitted on 1 Jan 1979

HAL is a multi-disciplinary open access archive for the deposit and dissemination of scientific research documents, whether they are published or not. The documents may come from teaching and research institutions in France or abroad, or from public or private research centers.
L'archive ouverte pluridisciplinaire HAL, est destinée au dépôt et à la diffusion de documents scientifiques de niveau recherche, publiés ou non, émanant des établissements d'enseignement et de recherche français ou étrangers, des laboratoires publics ou privés. 


\title{
LE JOURNAL DE PHYSIQUE-LETTRES
}

Classification

Physics Abstracts

$23.60-21.60 \mathrm{E}$

\section{La radioactivité alpha étudiée par les méthodes de la fission}

\author{
D. N. Poenaru $\left({ }^{*}\right)$, M. Ivaşcu $\left({ }^{*}\right)$ et A. Săndulescu $\left({ }^{* *}\right)$ \\ (*) Institut de Physique et d'Ingénierie Nucléaire, B.P. 5206, R-76900 Bucarest, Roumanie. \\ (**) Institut für Theoretische Physik der Universität Frankfurt am Main, R.F.A.
}

(Reçu le 14 mai 1979, révisé le 25 juin 1979, accepté le 23 juillet 1979)

\begin{abstract}
Résumé. - L'énergie potentielle du système alpha-noyau est calculée en utilisant le modèle de la goutte liquide avec une correction des couches phénoménologiques permettant de reproduire exactement $Q_{\alpha}$ expérimental. Les durées de vie théoriques des 58 émetteurs ( $\mathrm{Po}, \mathrm{Ra}, \mathrm{Th}, \mathrm{U}, \mathrm{Pu}, \mathrm{Cm}, \mathrm{Cf}, \mathrm{Fm}$ et $\mathrm{No}$ ) sont en bon accord avec l'expérience, suggérant que la désintégration alpha peut être considérée comme un processus de fission.
\end{abstract}

\begin{abstract}
Alpha-nucleus potential energy is computed by using the liquid drop model with a phenomenological shell correction allowing to reproduce exactly the experimental $Q_{\alpha}$-values. Theoretical half lives of 58 emitters ( $\mathrm{Po}, \mathrm{Ra}, \mathrm{Th}, \mathrm{U}, \mathrm{Pu}, \mathrm{Cm}, \mathrm{Cf}, \mathrm{Fm}$ and $\mathrm{No}$ ) are in good agreement with experimental ones suggesting that alpha decay could be interpreted as a spontaneous fission process.
\end{abstract}

Le modèle de la goutte liquide [1] complété par les corrections de couches $[2,3]$ est souvent appliqué à l'étude de la fission des noyaux [4] pour calculer l'énergie potentielle du système. La durée de vie est trouvée [3] avec la formule de la théorie W.K.B. Le but de cet article est de montrer que ces méthodes peuvent être appliquées aussi à l'étude de la désintégration alpha.

Nous considérons une paramétrisation simple de la surface nucléaire [5] : deux sphères avec les rayons $R_{1}, R_{2}$ et une distance des centres $-R$. L'origine de l'axe de symétrie $O_{z}$ est placée au centre de la grande sphère coupée (pour $R<R_{\mathfrak{t}}$ ) par le plan d'intersection des deux sphères $z=z_{\mathrm{c}}$. Le rayon $R_{2}$ de la calotte sphérique de volume $V_{2}$ qui représente le fragment alpha reste constant pendant la déformation, à partir du moment initial quand $R=R_{\mathrm{i}} \equiv R_{0}-R_{2}$. $R_{0}=r_{0} A^{1 / 3}$ est le rayon du noyau-père. Au cours du temps, le volume $V_{2}$ et le rayon $R_{1}$ varient de manière à conserver le volume total du système. Jusqu'à $R=R_{\mathrm{i}}+0,7\left(R_{\mathrm{t}}-R_{\mathrm{i}}\right)$ où $R_{\mathrm{t}}=R_{1 \mathrm{f}}+R_{2}$ et $R_{1 \mathrm{f}}$ est le rayon du noyau-fille, on considère que la densité de charge des deux fragments est la même. Après quoi, le numéro de charge $Z_{2}$ du petit fragment varie linéairement en $R$ jusqu'à $R=R_{\mathrm{t}}$. Les termes de surface $E_{\mathrm{s}}$, et coulombien $E_{\mathrm{c}}$ de l'énergie de la goutte liquide

$$
E_{\mathrm{LD}}=E_{\mathrm{s}}+E_{\mathrm{c}}+E_{V}-E^{\infty}
$$

sont calculés avec le programme [6] modifié pour tenir compte de la densité de charge différente [5] :

$$
\begin{gathered}
E_{\mathrm{s}}=E_{\mathrm{s} 1}+E_{\mathrm{s} 2} \\
E_{\mathrm{s} i}=a_{\mathrm{s}}\left[1-\kappa\left(N_{i}-Z_{i}\right)^{2} / A_{i}^{2}\right] A_{i}^{2 / 3} S_{i} /\left(4 \pi R_{0}^{2}\right) \\
(i=1,2) \\
E_{\mathrm{c}}=\frac{\rho_{1 e}^{2}}{2} \int_{V_{1}} \mathrm{~d}^{3} r_{1} \int_{V_{1}} \frac{\mathrm{d}^{3} r_{2}}{r_{12}}+\frac{\rho_{2 e}^{2}}{2} \int_{V_{2}} \mathrm{~d}^{3} r_{1} \int_{V_{2}} \frac{\mathrm{d}^{3} r_{2}}{r_{12}}+ \\
+\rho_{1 e} \rho_{2 e} \int_{V_{1}} \mathrm{~d}^{3} r_{1} \int_{V_{2}} \frac{\mathrm{d}^{3} r_{2}}{r_{12}} \\
E_{V}=E_{V 1}+E_{V 2} ; \\
E_{V i}=-a_{V}\left[1-\kappa\left(N_{i}-Z_{i}\right)^{2} / A_{i}^{2}\right] A_{i}
\end{gathered}
$$

où $r_{12}=\left|\mathbf{r}_{1}-\mathbf{r}_{2}\right| ; a_{\mathrm{s}}=17,9439 \mathrm{MeV} ; \kappa=1,7826$; $r=1,2249 \mathrm{fm} ; a_{V}=15,4941 \mathrm{MeV} ; N_{i}=A_{i}-Z_{i}$; $A_{2}=4 V_{2} / V_{2 \mathrm{f}} ; A_{1}=A-A_{2} ; S_{i}$ est l'aire de la surface extérieure et $\rho_{i e}$ la densité de charge du fragment $i$. Il est évident que pour $R=R_{\mathrm{i}}$, quand $V_{2}=0$ on retrouve l'énergie du noyau-père : $E_{\mathrm{s}}\left(R_{\mathrm{i}}\right)=E_{\mathrm{s}}^{0}$; $E_{\mathrm{c}}\left(R_{\mathrm{i}}\right)=E_{\mathrm{c}}^{0} ; E_{V}\left(R_{\mathrm{i}}\right)=E_{V}^{0}$. $E_{V}$ est l'énergie de volume [1] et $E=E_{1}+E_{2}$ est la somme des énergies propres des deux fragments séparés. Grâce au choix particulier de l'origine d'énergie, on obtient à $R=R_{\mathrm{i}}$ la valeur théorique de la chaleur de réaction

$$
Q_{\mathrm{th}}=\left.E_{\mathrm{LD}}(R)\right|_{R=R_{\mathrm{i}}},
$$


et à partir de la scission $\left(R \geqslant R_{\mathrm{t}}\right)$

$$
E_{\mathrm{LD}}=Z_{1} Z_{2} e^{2} / R \text {. }
$$

La figure 1 montre les différences entre les valeurs expérimentales $Q_{\exp }$ [7] et théoriques des $Q_{\alpha}$. On remarque un écart moyen provenant du fait qu'on ignore d'autres termes de la formule de masse [1].

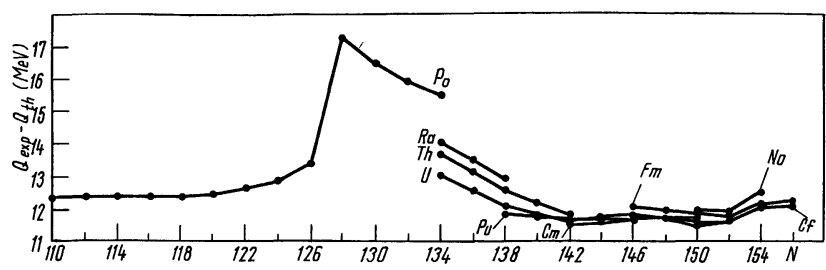

Fig. 1. - Différences entre les valeurs expérimentales et théoriques des $Q_{\alpha}$ en fonction du nombre des neutrons $N$ pour divers noyaux pair-pair.

[Differences between experimental and theoretical $Q_{\alpha}$ - values $v s$. neutron number $N$ for varıous even-even nuclei.]

Par ailleurs les effets de couches apparaissent clairement autour du nombre des neutrons $126 \mathrm{du}$ noyaufille - doublement magique, qui correspond à $N=128$. Malheureusement, on ne dispose pas d'une méthode de calcul microscopique des corrections de couches pour une asymétrie très grande. Pour reproduire exactement les valeurs expérimentales de $Q_{\alpha}$, nous avons utilisé une correction empirique :

$$
\begin{aligned}
E(R) & =E_{\mathrm{LD}}(R)+E_{\mathrm{cor}}(R), \quad R<R_{\mathrm{t}} \\
E_{\mathrm{cor}} & =\left(Q_{\text {exp }}-Q_{\mathrm{th}}\right)\left[1-V_{2}(R) / V_{2 \mathrm{f}}\right]
\end{aligned}
$$

où $V_{2}$ est le volume du petit fragment et $V_{2 \mathrm{f}}=V_{2}\left(R_{\mathrm{t}}\right)$.

La durée de vie $T_{1 / 2}$, en secondes, est calculée [3] par la relation

$$
T_{1 / 2}=\ln 2 /(n P)=\left(1,4333 \times 10^{-21} / E_{\mathrm{vib}}\right) \exp (K)
$$

où $n=\omega / 2 \pi$ est le nombre d'assauts de la barrière par unité de temps en corrélation avec l'excitation $E_{\text {vib }}=\hbar \omega / 2$ [8] due aux vibrations de la surface et $P=\exp (-K)$ est la probabilité de pénétration donnée par la formule de W.K.B. Après avoir fait les substitutions des constantes numériques

$K=0,4392 \int_{R_{\mathrm{a}}}^{R_{\mathrm{b}}}\left\{\left[E(R)-E^{\prime}\right] A_{1} A_{2} / A\right\}^{1 / 2} \mathrm{~d} R$

les longueurs $R, R_{\mathrm{a}}, R_{\mathrm{b}}$ étant exprimées en fm et les énergies $E, E^{\prime}$ en $\mathrm{MeV} ; E^{\prime}=Q_{\exp }+E_{\text {vib }}$. Nous avons utilisé la valeur $E_{\text {vib }}=0,37 \mathrm{MeV}$, pour obtenir le meilleur accord avec l'expérience. $\left(A_{1} A_{2} / A\right) m=\mu$ est la masse réduite du système; $m$ est la masse d'un nucléon. $R_{\mathrm{a}}$ est obtenu par interpolation en utilisant l'expression $E\left(R_{\mathrm{a}}\right)=E^{\prime}$ et

$$
R_{\mathrm{b}}=Z_{1} Z_{2} e^{2} / E^{\prime} .
$$

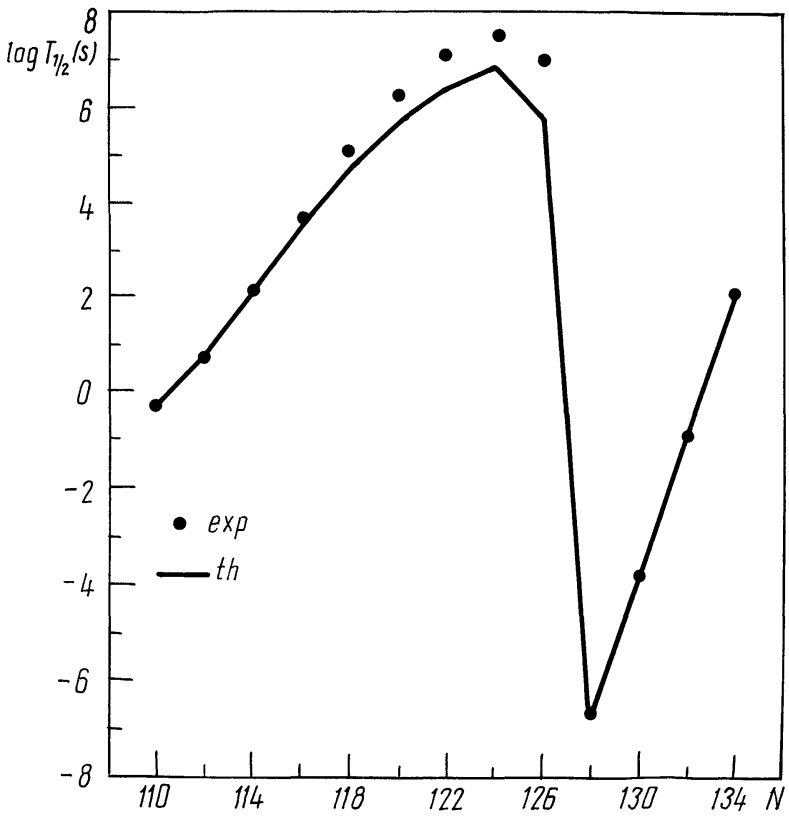

Fig. 2. - Comparaison de la durée de vie théorique avec la valeur expérimentale pour quelques isotopes du Po. $N$ est le nombre des neutrons du noyau père.

[Comparison on theoretical half-life with the experimental one for some isotopes of Po. $N$ is the neutron number of the parent nucleus.]

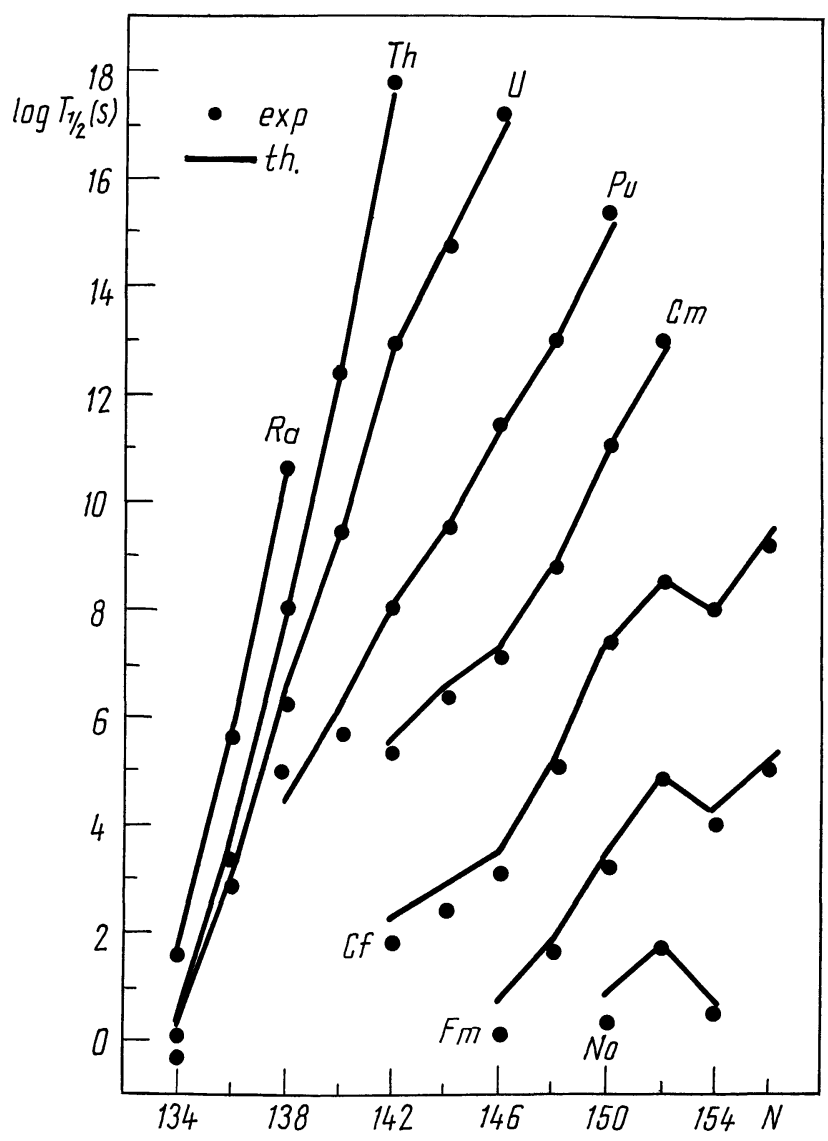

Fig. 3. - Mêmes résultats que sur la figure 2 pour $R a, T h, U$, $\mathrm{Pu}, \mathrm{Cm}, \mathrm{Cf}, \mathrm{Fm}$ et No.

[Same results as in figure 2 for $\mathrm{Ra}, \mathrm{Th}, \mathrm{U}, \mathrm{Pu}, \mathrm{Cm}, \mathrm{Cf}, \mathrm{Fm}$ and No.] 
L'intégrale (8) est calculée par la méthode de GaussLegendre. La durée moyenne du programme complet pour le calcul de la période d'un noyau donnée sur IBM $370 / 135$ est de $45 \mathrm{~s}$.

Les figures 2 et 3 montrent le bon accord de cette théorie avec les données expérimentales $[9,10]$ suggé- rant que la désintégration alpha peut être considérée comme un processus de fission très asymétrique.

Des résultats similaires ont été obtenus [11] avec les modèles de Krappe-Nix [12] et Krappe-NixSierk [13], qui remplacent l'énergie de surface $E_{\mathrm{s}}$ par une intégrale de convolution Yukawa ou Yukawa - plus - exponentielle.

\section{Bibliographie}

[1] Myers, W. D. and SwiateCKI, W. J., Nucl. Phys. 81 (1966) 1 ; Ark. Fys. 36 (1967) 343.

[2] Strutinsky, V. M., Nucl. Phys. A 95 (1967) 420.

[3] Brack, M., Damgaard, J., Jensen, A. S., Pauli, H. C., Strutinsky, V. M. and Wong, C. Y., Rev. Mod. Phys. 44 (1972) 320.

[4] Michaudon, A., Nuclear Fission (in Advances in Nucl. Phys., eds. M. Baranger, E. Voigt) (Plenum Press, New York) 1973, vol. 6.

[5] Poenaru, D. N., Mazilu, D. and Ivascu, M., J. Phys. G. : Nucl. Phys. (to be published).

[6] Poenaru, D. N. and Ivascu, M., Comput. Phys. Commun. 16 (1978) 85 .
[7] Wapstra, A. H. and Bos, K., At. Data Nucl. Data Tables 19 (1977) 177.

[8] Johansson, T., Nilsson, S. G. and Szymanski, Z., Ann. Phys. (Paris) 5 (1970) 377.

[9] Walen, R. J., C. R. Hebd. Séan. Acad. Sci. (Paris) 255 (1962) 1604.

[10] Rytz, A., At. Data Nucl. Data Tables 12 (1973) 479.

[11] Poenaru, D. N., Ivascu, M. and Sandulescu, A. (to be published).

[12] Krappe, H. J. and Nix, J. R., Proc. Third Int. Atomic En. Symp. on Physics and Chemistry of Fission, Rochester 1973 (IAEA, Vienna) 1974, Vol. I, p. 159.

[13] Krappe, H. J., Nix, J. R. and Sierk, A. J., Phys. Rev. Lett. 42 (1979) 215. 